\title{
Publisher Correction: High irradiance performance of metal halide perovskites for concentrator photovoltaics
}

Zhiping Wang (1D, Qianqian Lin (1D, Bernard Wenger, M. Greyson Christoforo, Yen-Hung Lin (D, Matthew T. Klug, Michael B. Johnston (1), Laura M. Herz (1) and Henry J. Snaith (1)

Correction to: Nature Energy https://doi.org/10.1038/s41560-018-0220-2, published online 6 August 2018.

When this Article was originally published, an old version of the associated Supplementary Information file was uploaded. This has now been replaced.

Published online: 13 August 2018

https://doi.org/10.1038/s41560-018-0238-5 